ARTICLE

\title{
Design of highly efficient deep-blue organic afterglow through guest sensitization and matrices rigidification
}

\author{
Shen Xu (1] ${ }^{1,4}$, Wu Wang ${ }^{1,4}$, Hui Li ${ }^{1}$, Jingyu Zhang ${ }^{1}$, Runfeng Chen (i] ${ }^{1 凶}$, Shuang Wang ${ }^{1}$, Chao Zheng ${ }^{1}$,
} Guichuan Xing (i) ${ }^{2 凶}$, Chunyuan Song (1) ${ }^{1} \&$ Wei Huang (i) ${ }^{1,3 凶}$

Blue/deep-blue emission is crucial for organic optoelectronics but remains a formidable challenge in organic afterglow due to the difficulties in populating and stabilizing the highenergy triplet excited states. Here, a facile strategy to realize the efficient deep-blue organic afterglow is proposed via host molecules to sensitize the triplet exciton population of guest and water implement to suppress the non-radiative decays by matrices rigidification. A series of highly luminescent deep-blue $(405-428 \mathrm{~nm})$ organic afterglow materials with lifetimes up to $1.67 \mathrm{~s}$ and quantum yields of $46.1 \%$ are developed. With these high-performance water-responsive materials, lifetime-encrypted rewritable paper has been constructed for water-jet printing of high-resolution anti-counterfeiting patterns that can retain for a long time (>1 month) and be erased by dimethyl sulfoxide vapor in 15 min with high reversibility for many write/erase cycles. These results provide a foundation for the design of highefficient blue/deep-blue organic afterglow and stimuli-responsive materials with remarkable applications.

\footnotetext{
${ }^{1}$ Key Laboratory for Organic Electronics and Information Displays \& Jiangsu Key Laboratory for Biosensors, Institute of Advanced Materials (IAM), Jiangsu National Synergistic Innovation Center for Advanced Materials (SICAM), Nanjing University of Posts and Telecommunications, 9 Wenyuan Road, Nanjing 210023, People's Republic of China. ${ }^{2}$ Institute of Applied Physics and Materials Engineering, University of Macau, Macao SAR 999078 , China. ${ }^{3}$ Shaanxi Institute of Flexible Electronics (SIFE), Northwestern Polytechnical University (NPU), 127 West Youyi Road, Xi'an 710072, People's Republic of China.

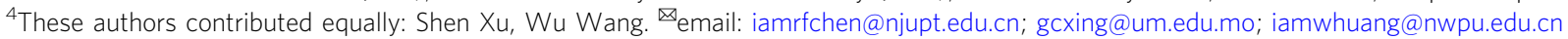


B lue luminescence, which has long been documented as a prime challenge in both fluorescent and phosphorescent materials $s^{1,2}$, is the core elements indispensable for solidstate lighting and full-color display technologies in organic optoelectronics $^{3-5}$. Organic ultralong room temperature phosphorescence (OURTP) with luminescent lifetime over $0.1 \mathrm{~s}$ after removing the excitation source has attracted considerable attention due to the fundamental breakthrough of the excited state lifetime tuning for organic afterglow with unique photophysical properties and innovative applications in many fields 6,7 . However, despite of the high molecular diversity of organic molecules, most organic afterglow emission bands are in a range from 500 to $600 \mathrm{~nm}$ owing to its triplet state and solid state luminescence nature; both of them will lead to low-lying exciton energies and bathochromic shifts of the emission spectrum ${ }^{8,9}$. It becomes even more challenging to develop blue OURTP, which should populate and stabilize the high-lying triplet excited state simultaneously 9,10 .

Crystallization/H-aggregation ${ }^{11,12}$ and exciplex formation ${ }^{13-15}$ strategies previously proposed to construct OURTP are difficult to realize the blue organic afterglow owing to the unavoidable red-shift compared to the single-molecular fluorescence after the aggregation coupling and intermolecular electronic interaction in solid states ${ }^{16}$. Dispersing emitters in host is effective in preventing the bathochromic shift and eliminating the concentration quenching by inhibiting molecular aggregation and electronic coupling at low doping concentrations ${ }^{17}$. To achieve highly efficient OURTP, rigid host matrixes are essentially needed to suppress the non-radiative relaxation decays for high phosphorescent quantum yield (PhQY) ${ }^{18,19}$. Nevertheless, rigid host molecules with strong intermolecular interactions are generally hard to be processed and the single molecular dispersion of the dopant suffers from the low compatibility between the highly polar host and luminescent guest ${ }^{20}$. Moreover, the luminance of the hostguest material is usually weak due to the small amount of the doped emitters in the optically inert host. Therefore, despite the recent efforts ${ }^{21,22}$, it remains difficult to achieve efficient blue/ deep-blue organic afterglow with long lifetime $(>1.0 \mathrm{~s})$ and high PhQY (>40\%).

Here, we propose a general strategy to overcome these intrinsic difficulties in designing blue organic afterglow using active host for triplet excited state sensitization and water implement for matrices rigidification to simultaneously elongate the lifetime and boost the PhQY of OURTP. Guest sensitization through efficient energy transfer from host to guest promotes significantly the excitation of the small amount $(\sim 0.5 \%)$ of doped guest molecules for strong OURTP. Matrices rigidification using water, inspired by the solidification process of concrete ${ }^{23}$, greatly rigidifies the host-guest system by forming hydrogen-bonding (H-bonding) networks and this post-rigidification approach makes the rigid hosts not obligated, which should significantly facilitate the material design and preparation of high-performance organic afterglow materials. Indeed, a series of deep-blue organic afterglow materials are achieved and the OURTP lifetimes are improved up to $1.67 \mathrm{~s}$ and PhQY to $46.1 \%$, which are among the best organic afterglow performance reported so $\mathrm{far}^{7,8}$. Further, in light of the extraordinary water-responsible OURTP, rewritable lifetime-encryption paper for anticounterfeiting is constructed. High-resolution patterns can be printed by a commercial ink-jet printer using pure water as ink and eliminated by dimethyl sulfoxide (DMSO) vapor fuming with high reversibility.

\section{Results}

Material design and preparation. The deep-blue OURTP materials were prepared by using cyanuric acid (CA) as a universal host owing to its abundant interaction positions that can efficiently suppress the luminescence quenching effects and non-radiative decay processes, and phthalic acid derivatives as the guest molecules because they can interact effectively with CA for single molecular dispersion and suppressed non-radiative vibration through $\mathrm{H}$-bonding (Fig. 1a) ${ }^{24,25}$. Moreover, CA has very high lowest singlet $\left(S_{1}\right)$ and triplet $\left(T_{1}\right)$ excited state energies ${ }^{26}$ to support efficient energy transfer and function as active host to sensitize the triplet excited state of the guest. CA also has high optical inertness ${ }^{27}$ for both photoexcitation and luminescence extraction of guest's emission. Specifically, the unique cyclic amide structure of CA contains three $\mathrm{N}$ atoms and three carbonyl groups with lone-pair-electrons to facilitate the spin-forbidden intersystem crossing (ISC) for facile population of $\mathrm{T}_{1}$ state as well as three $\mathrm{H}$-bond donor and three $\mathrm{H}$-bond acceptor positions for constructing $\mathrm{H}$-bonding cross-linked networks with other molecules to efficiently suppress non-radiative decay channels. Trimesic acid (TMA) with three carboxyl moieties which can facilitate the $n-\pi^{*}$ transition, spin-orbit coupling, and molecular interactions with CA is chosen as the guest molecule (Supplementary Fig. 1). It should be noted that TMA crystal shows OURTP with lifetime of $0.15 \mathrm{~s}$ and PhQY of $2.7 \%$ at $524 \mathrm{~nm}$, suggesting its intrinsic OURTP nature without the help of other materials ${ }^{28}$. After being doped into CA at a low concentration of $5 \mathrm{wt} \%$ via simply ultrasonicating aqueous mixture of TMA and $\mathrm{CA}$ at room temperature (Fig. 1b), the as-prepared powder of CT5-0 after removing the solvent under vacuum at $40^{\circ} \mathrm{C}$ for $24 \mathrm{~h}$ exhibits long phosphorescent lifetime of $1.13 \mathrm{~s}$ and PhQY of $9.3 \%$ under ambient conditions (Fig. 1c). Interestingly, this efficient OURTP is deep blue $(406 \mathrm{~nm})$, which is very different to its intrinsic green emission. More interestingly, when $20 \mathrm{wt} \%$ water was added into, both phosphorescent lifetime and efficiency of the resulted powder (CT5-20) are significantly enhanced, reaching $1.67 \mathrm{~s}$ and $46.1 \%$, respectively (Fig. $1 \mathrm{~d}, \mathrm{e}$ ). To the best of our knowledge, both the lifetime and PhQY are among the highest ones of OURTP ${ }^{29-31}$, let alone the hardly available deep-blue organic afterglow (Supplementary Table 1, Supplementary Note 1 and Supplementary Figs. 2-4 ${ }^{26}$.

Photophysical properties of CT powders. To explore the extraordinary highly efficient deep-blue OURTP, the steady-state and time-resolved photophysical properties of CA, TMA, and CT5-0 were investigated $^{32}$. The optical bandgaps of CA and TMA are very large, showing strong absorption bands before $250 \mathrm{~nm}$ and fluorescence peaks around $300 \mathrm{~nm}$ (Fig. 2a and Supplementary Fig. 5). TMA also shows a decent phosphorescence band around $400 \mathrm{~nm}$ even at single molecular state in ethanol, while the steady-state photoluminescence (PL) spectrum of CA is dominated by the blue phosphorescence. Thus, both CA and TMA are capable of populating $\mathrm{T}_{1}$ upon photoexcitation for blue phosphorescence with weak brightness, low quantum efficiencies, and short lifetimes (Supplementary Table 2). But, more facile ISC were observed in CA with much weaker fluorescence in the steady-state PL spectrum (Fig. 2a) and larger spin-orbital coupling (SOC) constants from the Dalton calculations (Supplementary Table 3). Excitingly, when CA and TMA are mixed properly, the composite of CT5-0 exhibits almost identical phosphorescence spectrum to that of TMA (Supplementary Fig. 6) but with significantly increased PhQY and elongated lifetime. When water is implemented, this blue phosphorescence band further enhances and reaches the highest at $20 \mathrm{wt} \%$ water content (Fig. 2b, c, and Supplementary Fig. 7). The optimal TMA doping concentration is $5 \mathrm{wt} \%$ (Fig. $2 \mathrm{~d}$, e) and the emission band at $406 \mathrm{~nm}$ is nearly pure phosphorescence without the component of short-lived fluorescence (Fig. 2f, g, and Supplementary Fig. 8). In addition, although the afterglow intensity is highly dependent on 


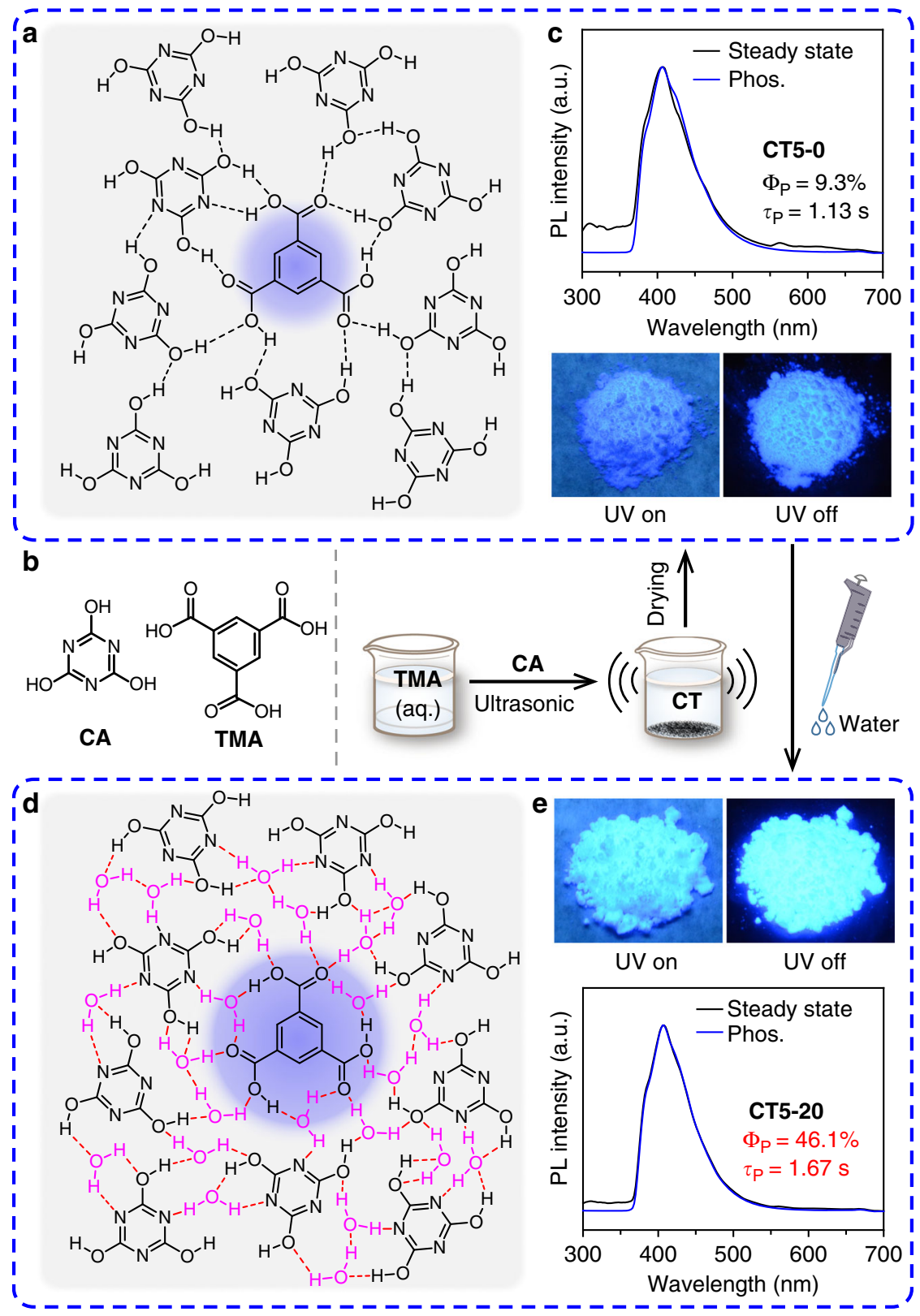

Fig. 1 Preparation of highly efficient deep-blue OURTP materials. a, $\mathbf{d}$ Molecular interactions between CA and TMA before (a) and after (d) the water implement. b Molecular structures of CA and TMA, and preparing procedures of their composites (CT). c, e Steady state and phosphorescent (phos.) spectra and photographs under (UV on) or after (UV off) $254 \mathrm{~nm}$ irradiation of CT5-0 (c) and CT5-20 (e).

water content and doping concentration, the afterglow lifetime is less sensitive to these variations, and nearly invariant under different doping levels of TMA, especially when water content is higher than $20 \mathrm{wt} \%$ (Fig. 2h, i, and Supplementary Fig. 9). Therefore, the CT composite reaches the best performance at $5 \mathrm{wt} \%$ TMA doping and $20 \mathrm{wt} \%$ water implement, exhibiting the strongest blue OURTP excitable from 210 to $300 \mathrm{~nm}$ with lifetime up to $1.67 \mathrm{~s}$ (Fig. 2j and Supplementary Figs. 10, 11).

Mechanism of the efficient deep-blue OURTP. To gain further insight into the mechanism of the deep-blue OURTP, temperature-dependent PL measurements were performed. When temperature drops gradually from 278 to $78 \mathrm{~K}$, the phosphorescence spectrum of CT5-0 remains but the intensity increases constantly along with the elongated lifetime from 1.1 to $2.0 \mathrm{~s}$ (Fig. 3a and Supplementary Fig. 12). Similar behavior was observed in CT5-20 and CT5-40 (Fig. 3b and Supplementary Figs. 13, 14) but with limited phosphorescence intensity and lifetime enhancements in these water stimulated samples, suggesting that both low temperature and water implement can suppress the non-radiative decays for longer OURTP lifetime (Supplementary Table 4).

To understand the exact role of water in the composite, differential scanning calorimetry (DSC) measurements, Raman spectra, solid-state nuclear magnetic resonance (NMR) spectra 
a

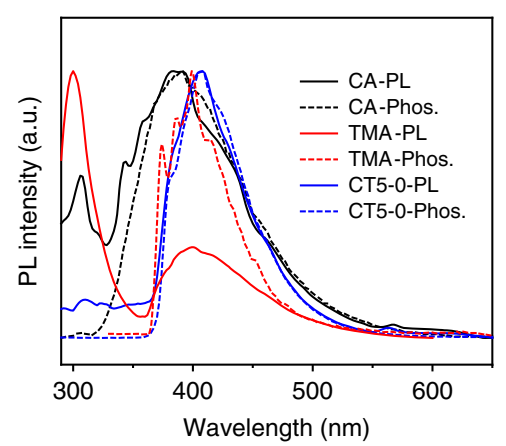

d

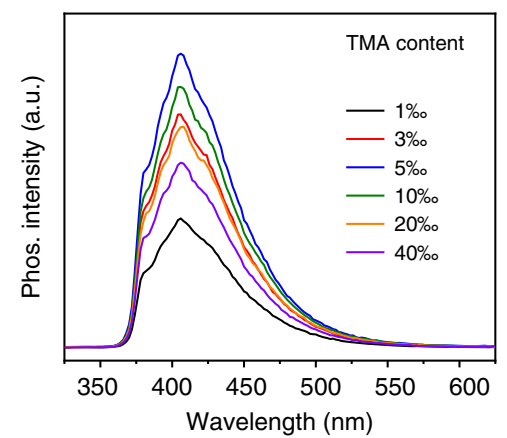

$\mathbf{9}$

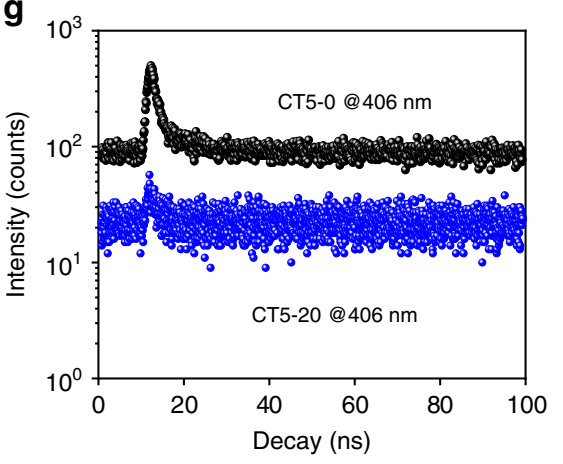

b

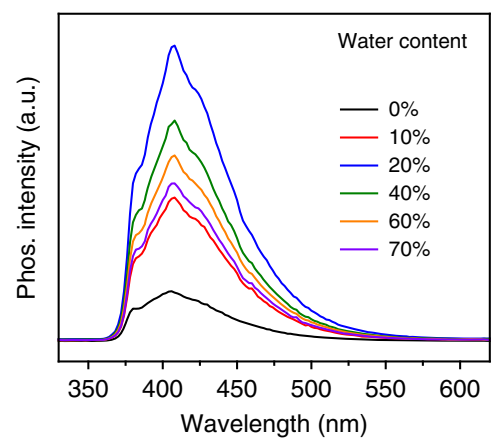

e

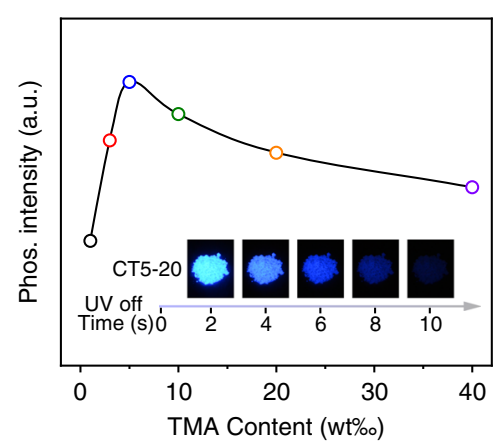

h
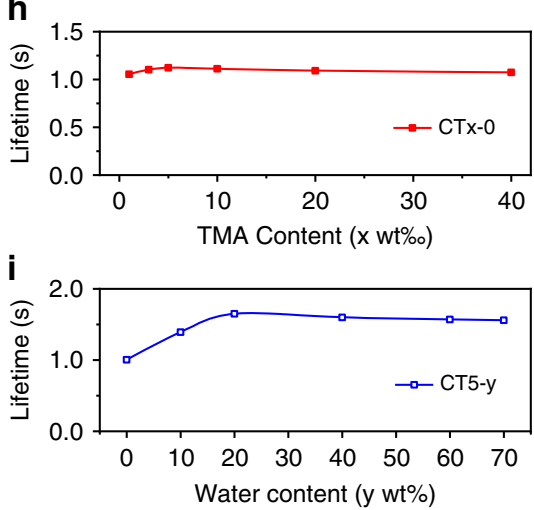

C

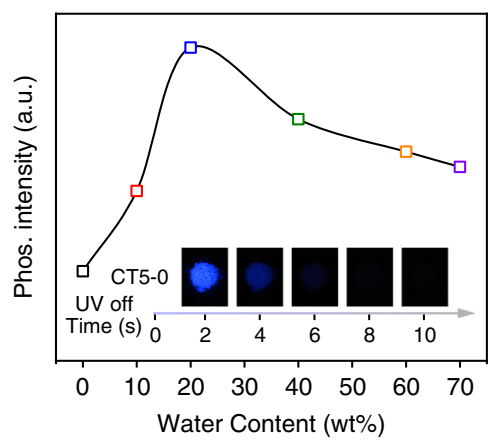

f

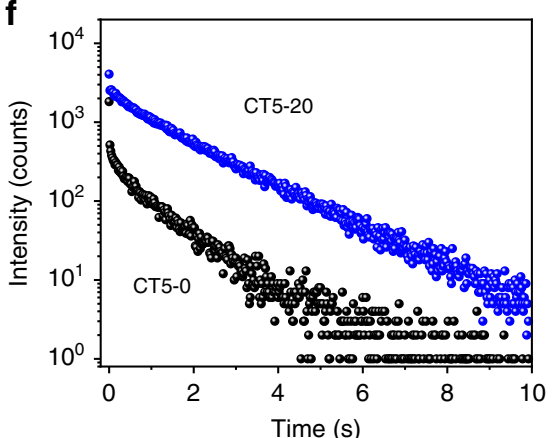

j

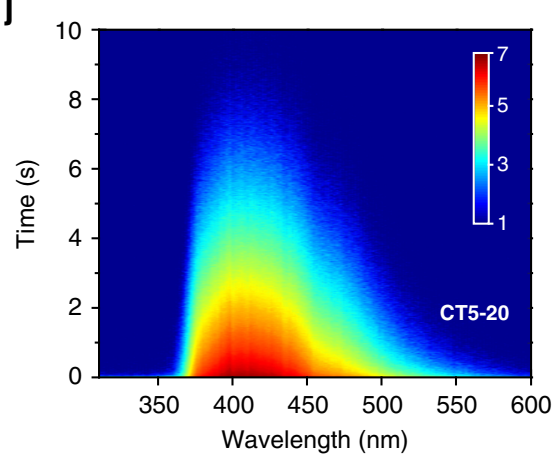

Fig. 2 Photophysical properties of CT powders at room temperature. a Steady-state PL (solid line) and phosphorescence (dash line, delay $30 \mathrm{~ms}$ ) spectra of CT5-0 powder at $298 \mathrm{~K}$ as well as TMA in ethanol $(10 \mu \mathrm{M})$ and CA powder at $77 \mathrm{~K}$. b-e Phosphorescent spectra (b, d) and $406 \mathrm{~nm}$ emission intensities $(\mathbf{c}, \mathbf{e})$ of CT5-y with various water contents $(y$ wt $\%)(\mathbf{b}, \mathbf{c})$ and dry CTx-0 powder with various doping concentrations ( $x$ wt\%o) of TMA (d, e). Insets: photographs of CT5-0 and CT5-20 taken after removing the excitation of $254 \mathrm{~nm}$ UV light. $\mathbf{f}, \mathbf{g}$ OURTP (f) and fluorescence (g) decay curves of CT5-0 (black) and CT5-20 (blue) at $406 \mathrm{~nm}$. h, i OURTP lifetimes of CTx-0 with various TMA concentrations (h) and CT5-y with various water contents (i). $\mathbf{j}$ Time-resolved emission scanning spectrum of CT5-20. The excitation wavelength is $248 \mathrm{~nm}$.

and powder X-ray diffraction (XRD) of CT5 with different water contents were investigated. An endothermic melting peak around $7^{\circ} \mathrm{C}$ owing to the existence of the freezing bound water and a broad endothermic peak due to the continuous evaporation of water at high temperatures $\left(45-110^{\circ} \mathrm{C}\right)$ were observed in DSC curves when the water content reaches $40 \%$ (Fig. 3c) ${ }^{33}$. From the solid-state ${ }^{13} \mathrm{C}$-NMR spectra of CT5-0, there is a splitting $(152.3$ and $149.8 \mathrm{ppm}$ ) of the carbon atom of CA at $150.4 \mathrm{ppm}$ (Supplementary Fig. 15 and Supplementary Note 2), indicating the partly $\mathrm{H}$-bonding of the host molecule with or without $\mathrm{H}$ bonding (Fig. 3d) ${ }^{24}$. This splitting disappears after water implement, suggesting that all the polar moieties were strengthened by the H-bonds formed among CA, TMA and water (Fig. 1d). The insertion of water for constructing water-inducted $\mathrm{H}$-bonds will lead to the expanding of the composites, which was observed in the XRD curves with reduced $2 \theta$ due to the increased interplanar distance of CA after water implement (Fig. 3e).
Further, the H-bond formation was confirmed by the Raman spectra, showing clear shifts of the 1725 and $701 \mathrm{~cm}^{-1}$ bands after water addition owing to the intermolecular interactions between the $\mathrm{C}=\mathrm{O}$ of $\mathrm{CA}$ and water molecules, no matter whether doping TMA or not (Fig. 3f). Therefore, an excellent H-bonding network is formed after the introduction the third component of water, resulting in significantly rigidified matrices of the composites with much suppressed non-radiative decays and reduced penetration of oxygen and its quenching effects for greatly improved OURTP performance. Furthermore, H-bonds is beneficial to enhance ISC and Dexter energy transfer ${ }^{34,35}$, contributing further to the increased phosphorescence intensity and PhQY after water implement. It should be noted that when other solvents were added to the CT5-0 powder, the phosphorescence intensity and lifetime were generally reduced, confirming that only water can play this bridging role here in forming $\mathrm{H}$-bonds for the matrices rigidification (Supplementary Fig. 16). In addition, the $\mathrm{pH}$ can 
a
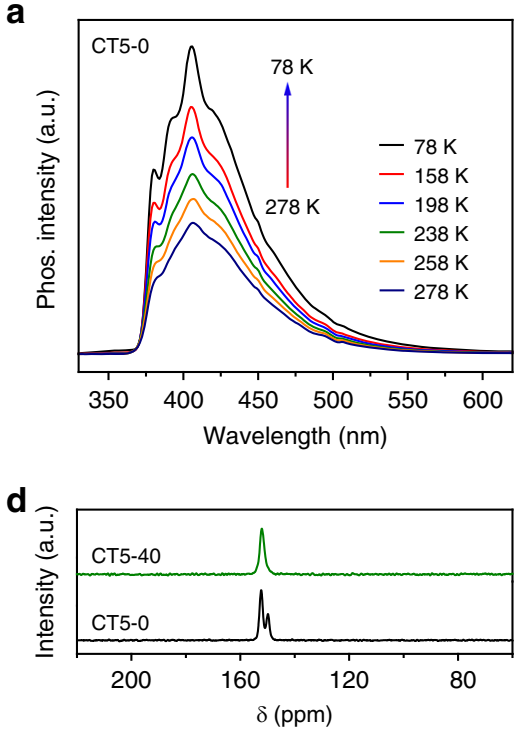

e
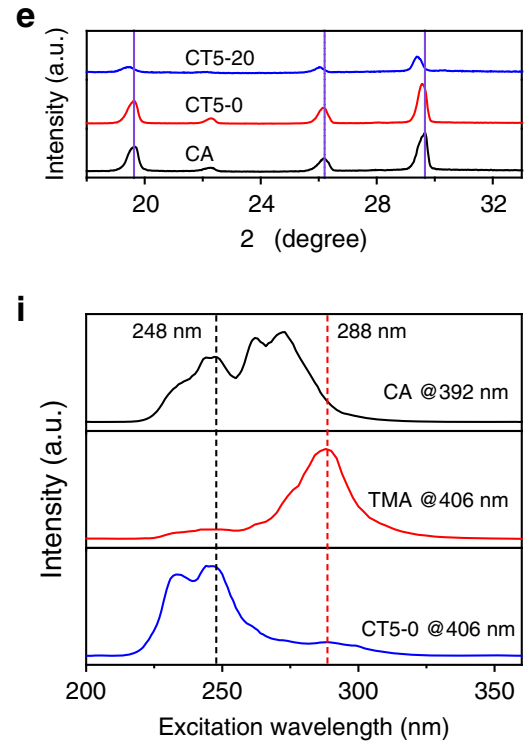

b

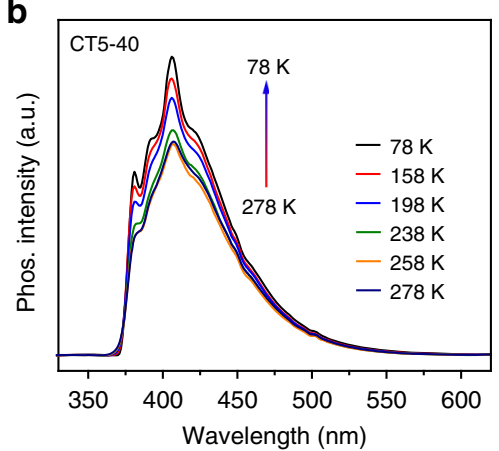

f
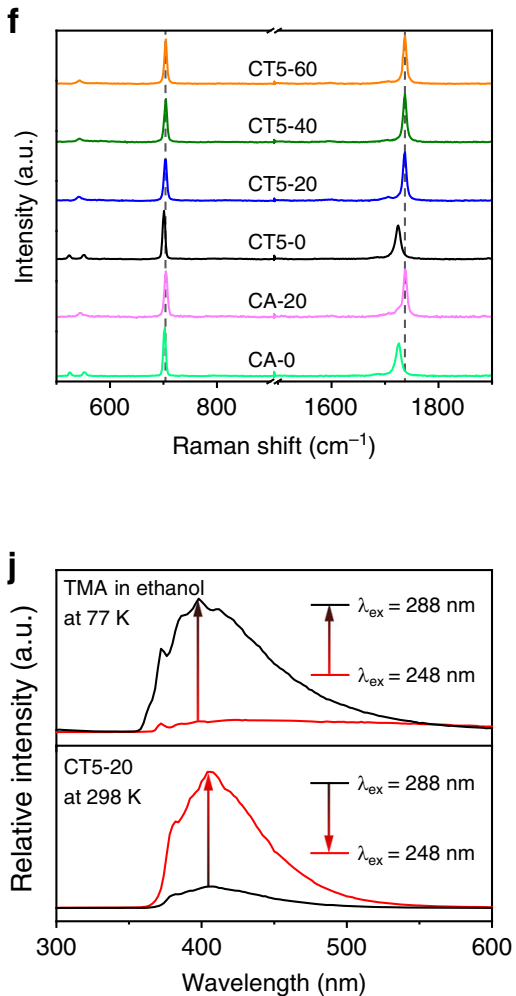

C

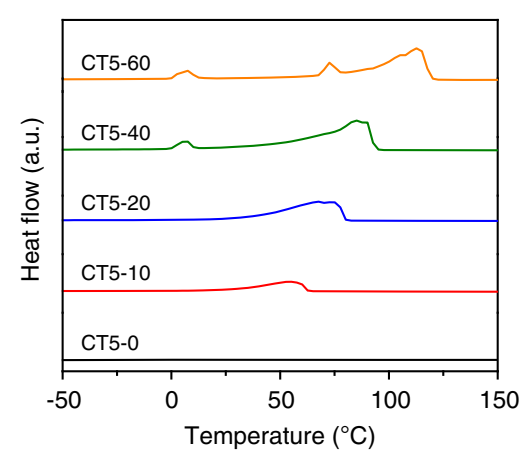

g

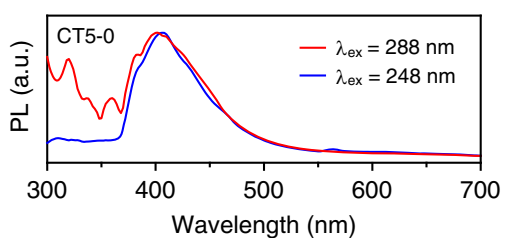

h

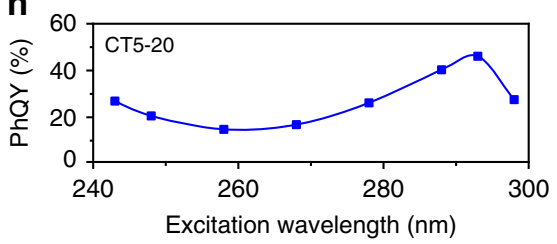

k

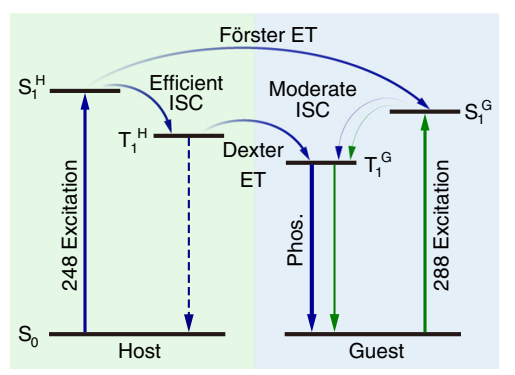

Fig. 3 Mechanism in realizing the highly efficient deep-blue OURTP. a, b Temperature-dependent phosphorescence spectra of CT5-0 (a) and CT5-40 (b) from 278 to $77 \mathrm{~K}$ excited at $248 \mathrm{~nm}$. c DSC curves of CT5 with various water contents. d Solid state ${ }^{13} \mathrm{C}-\mathrm{NMR}$ spectra of CT5-40 and CT5-0. e XRD spectra of CA, CT5-0 and CT5-20. f Raman spectra of CA with 0 (CA-0) and 20 wt\% (CA-20) water and CT5 with various water content. $\mathbf{g}$ Steady-state PL spectra of CT5-0 excited by 248 (blue) and $288 \mathrm{~nm}$ (red) UV-light. $\mathbf{h}$ PhQY of CT5-20 at different excitation wavelength. $\mathbf{i}$ Excitation spectra of CA and CT5-0 powders and TMA in ethanol. $\mathbf{j}$ Phosphorescence spectra of TMA in ethanol at $77 \mathrm{~K}$ and CT5-20 at $298 \mathrm{~K}$ excited by 248 and $288 \mathrm{~nm}$ after $30 \mathrm{~ms}$ delay. $\mathbf{k}$ Proposed mechanism for the highly luminescent deep-blue OURTP.

also influence the OURTP intensity, and the strongest OURTP was observed at the original state when $\mathrm{pH}=2$ (Supplementary Fig. 17). Either increasing or reducing the $\mathrm{pH}$ will lead to lower OURTP, probably due to the difficulties in forming $\mathrm{H}$-bonds at other $\mathrm{pH}$ values ${ }^{27}$.

Besides the water-sensitive OURTP feature, the emission intensity of the CT composite is also dependent on the excitation wavelength. The almost invisible fluorescence of CT5- 0 excited by $248 \mathrm{~nm}$ can be apparently observed under excitation of $288 \mathrm{~nm}$ (Fig. 3g). And, the PhQY of CT5-20 reaches the highest value of $46.1 \%$ at $293 \mathrm{~nm}$ excitation with the same emission spectrum (Fig. $3 \mathrm{~h}$ and Supplementary Fig. 18). From the phosphorescence excitation spectra, CT5-0 has two excitation bands around 248 and $288 \mathrm{~nm}$ corresponding to the excitation of CA and TMA, respectively (Fig. 3i). When changing the excitation wavelength from 248 to 288 $\mathrm{nm}$, the phosphorescence intensity of pure TMA rises significantly, but that of CT5-20 decrease dramatically (Fig. 3j). This extraordinary phenomenon suggests that $\mathrm{CA}$ should act as an active host to sensitize TMA by energy transfer (ET) under $248 \mathrm{~nm}$ irradiation ${ }^{36}$. It is speculated that $248 \mathrm{~nm}$ UV-light excites the host material of CA to $\mathrm{S}_{1}{ }^{\mathrm{H}}$, which quickly transforms to $\mathrm{T}_{1}{ }^{\mathrm{H}}$ to populate $\mathrm{T}_{1}$ of the guest of TMA $\left(\mathrm{T}_{1}{ }^{\mathrm{G}}\right.$ ) by Dexter ET, or through Förster ET to form $\mathrm{S}_{1}{ }^{\mathrm{G}}$ and then $\mathrm{T}_{1}{ }^{\mathrm{G}}$ for the long-lived OURTP. The Dexter ET should be the main route during energy transfer from CA to TMA to sensitize $\mathrm{T}_{1} \mathrm{G}$, since the CA is an efficient phosphor with facile ISC for the dominated phosphorescence (Fig. 2a) and the fluorescence of TMA is negligible in the steady-state PL of CT5 composites excited at 248 $\mathrm{nm}$. When CT5 is excited by $288 \mathrm{~nm}$, TMA is directly excited to $\mathrm{S}_{1}{ }^{\mathrm{G}}$, which has moderated ISC to populate $\mathrm{T}_{1} \mathrm{G}_{\text {; }}$ therefore, both fluorescence and phosphorescence can be observed (Fig. 3g). Since the triplet excitons formation of TMA by CA sensitization under $248 \mathrm{~nm}$ excitation are much more efficient than that under $288 \mathrm{~nm}$ through direct TMA excitation, the OURTP intensity is much higher than the latter (Fig. 3j). But owing to fewer excited states involved, 
a

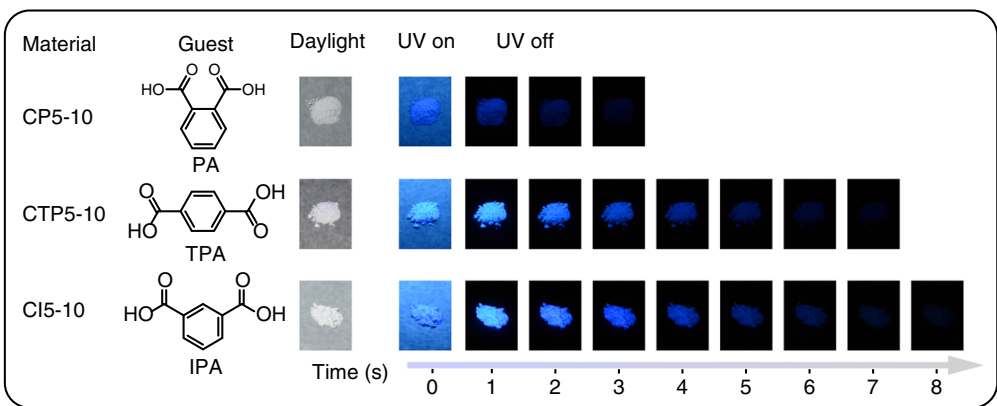

c

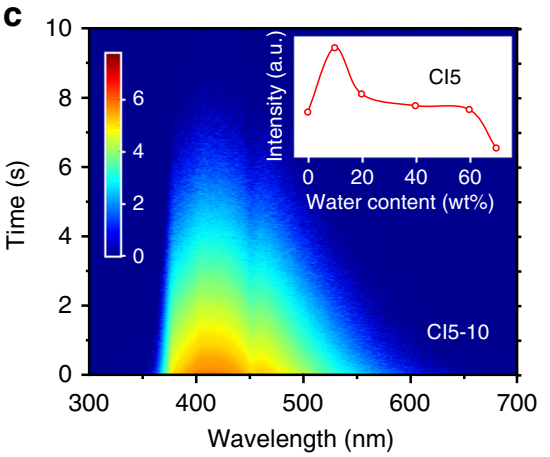

d

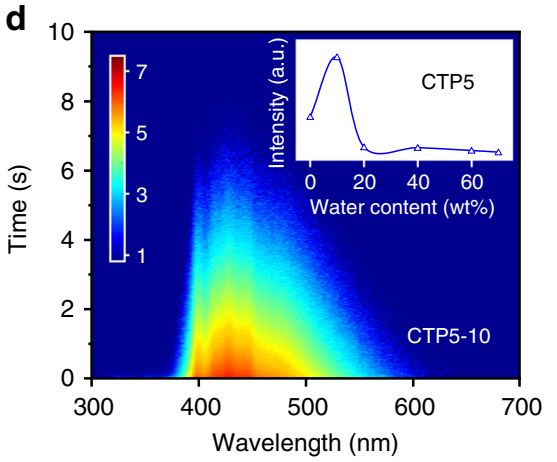

b

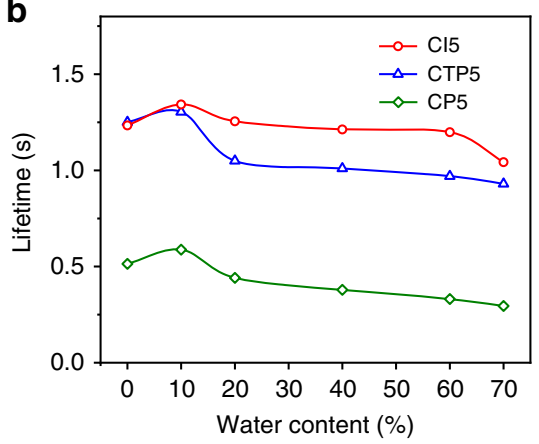

e

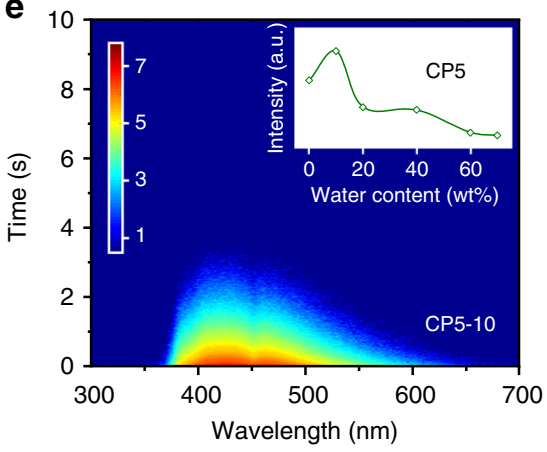

Fig. 4 Universality of the deep-blue OURTP design strategy. a Chemical structures of guest molecules and photographs of CI5-10, CTP5-10, and CP5-10

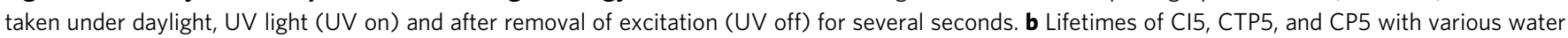
contents. c-e Time-resolved emission scanning spectra of CI5-10 (c), CTP5-10 (d), and CP5-10 (e). Insets: phosphorescence intensities of CI5, CTP5, and CP5 with various water contents.

higher PhQY can be achieved under $288 \mathrm{~nm}$ excitation (Fig. 3k). Taking together, the triplet sensitization by the active host and the matrices rigidification effect of water implement should be the two main factors in achieving the highly luminescent and efficient (46.1\%) deep-blue OURTP at room temperature.

Universality of the strategy. To verify the universality of this strategy using active host and water rigidification for designing high-performance blue/deep-blue organic afterglow materials, we tested other three widely studied benzoic acid derivatives of isophthalic acid (IPA), terephthalic acid (TPA), and phthalic acid (PA) as guest molecules (Fig. 4a) to construct the corresponding composites of CI, CTP, and CP (Supplementary Fig. 1 and Supplementary Table 5 ${ }^{28}$. Similarly, the doping in the active host of CA not only results in huge increase in lifetime up to $1.36 \mathrm{~s}$ and PhQY of $11.4 \%$, but also blue-shifts the emission bands to 405 , 428, and $425 \mathrm{~nm}$ for deep-blue OURTP (Supplementary Fig. 19). These OURTP spectra are also identical to the phosphorescent spectra of the guest molecules (Supplementary Fig. 20). Still, water-induced matrices rigidification for enhanced OURTP can be observed in these systems and the best water implement amount is $10 \mathrm{wt} \%$, possibly owing to the fewer H-bonding positions of the guest molecules (Fig. $4 \mathrm{~b}$ and Supplementary Fig. 21). This also leads to the best OURTP performance of CI5 (Fig. 4c-e) because IPA can form the good H-bonding network with CA, while the ortho-substituted PA has difficulties in interacting with host molecules to form $\mathrm{H}$-bonds to rigidify the matrices, resulting in the lowest performance of $\mathrm{CP} 5^{37}$. Besides, the fewer carboxyl groups of these benzoic acids leads to lower ISC rate and their fluorescence peaks can be observed in the steady-state spectra of these composites (Supplementary Fig. 22).

Water-jet rewritable encryption paper application. In light of the extraordinary water responsible OURTP behavior of these composites, rewritable paper which is crucial for environmental protection and sustainable development can be realized for waterjet printing ${ }^{38,39}$. Specifically, we coat CT5 solution $\left(100 \mathrm{mg} \cdot \mathrm{mL}^{-1}\right)$ in DMSO on a filter paper, followed by removing the solvent to obtain the encryption paper; this water-jet printed patterns can be facilely erased by DMSO vapor fuming at $120^{\circ} \mathrm{C}$ for $15 \mathrm{~min}$, representing the successful preparation of rewritable OURTP papers with lifetime-encryption for information storage and anticounterfeiting (Fig. 5a, Supplementary Fig. 23 and Supplementary Table 6). Other erasing solvents can also work, but DMSO is the most effective (Supplementary Fig. 24 and Supplementary Note 3). Along with the significantly enhanced OURTP after jetprinting using water as ink (Fig. 5b), this rewritable paper has excellent reversibility that can undergo many write/erase cycles (Fig. 5c and Supplementary Table 7). The strong and long-lived phosphorescence of the printed pattern can be conveniently captured by the camera of a mobile phone for the lifetimeresolved encryption of the printed patterns (Fig. 5d). The encrypted afterglow patterns are invisible under daylight and UV light, but can be easily observed after the removal of $254 \mathrm{~nm}$ UV irradiation after the repeated water-jet printing and DMSO vapor erasing cycles, presenting an excellent reusable anti-counterfeiting application of complex and high-resolution patterns (Fig. 5e and Supplementary Figs. 25, 26). Moreover, the patterns printed on the encryption paper are highly stable and can last more than 1 month under ambient conditions (Supplementary Fig. 27 and Supplementary Table 8). This deep-blue OURTP can be also used in white organic afterglow lighting with the aid of yellow organic afterglow materials (Supplementary Fig. 28 and Supplementary Note 4).

\section{Discussion}

In summary, we have established an effective approach to construct a series of heavy-atom-free deep-blue $(406-428 \mathrm{~nm})$ 
a

Preparation of rewritable paper

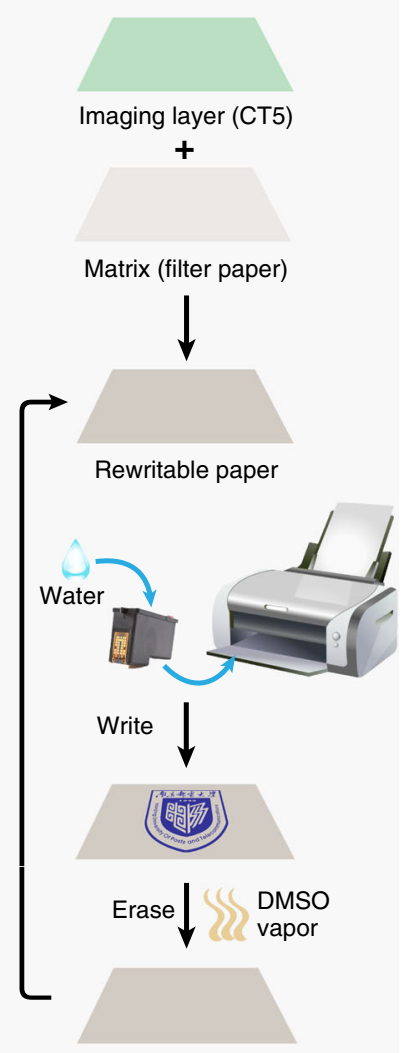

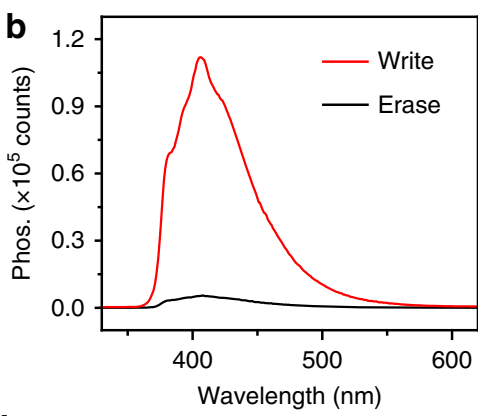
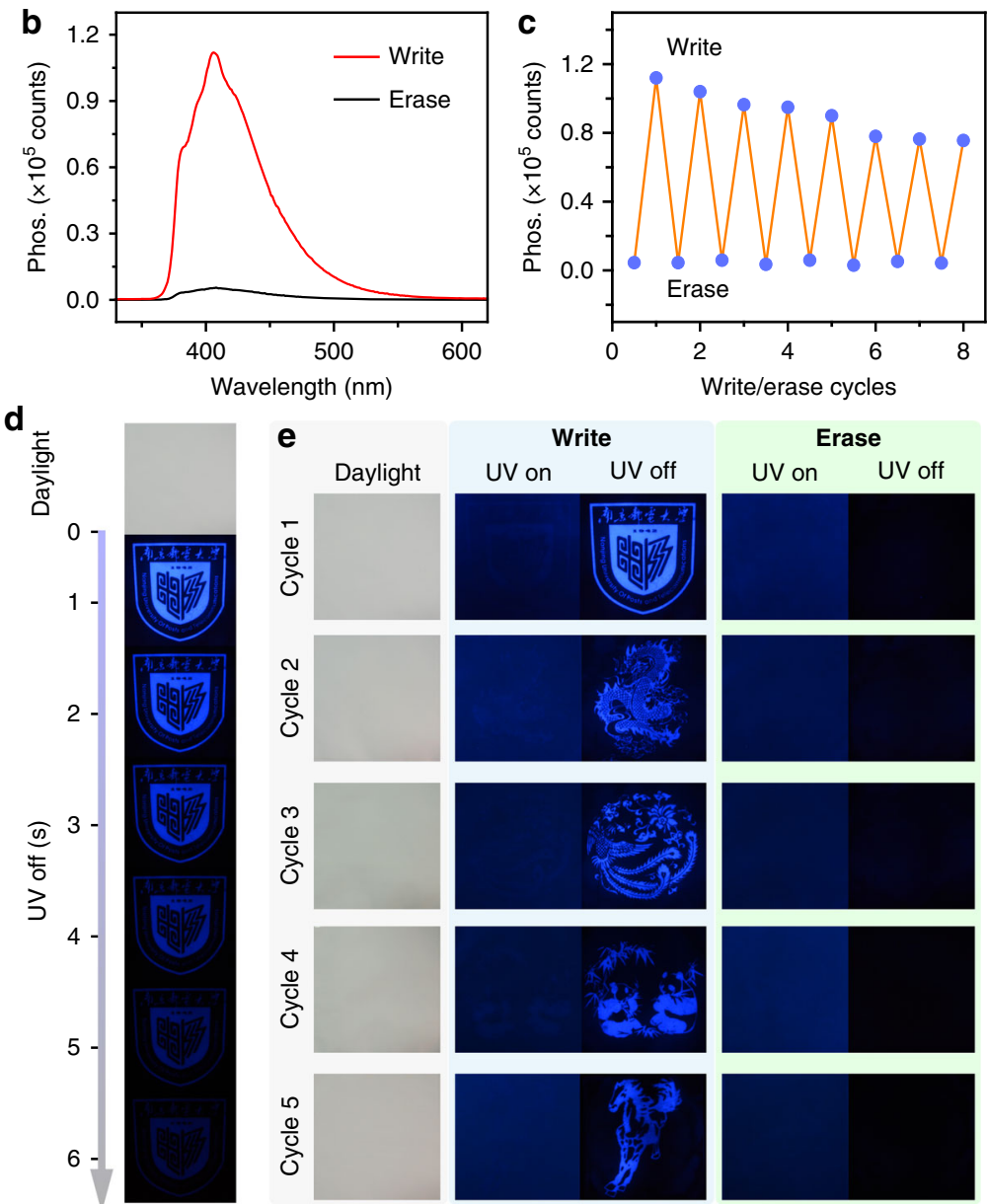

e
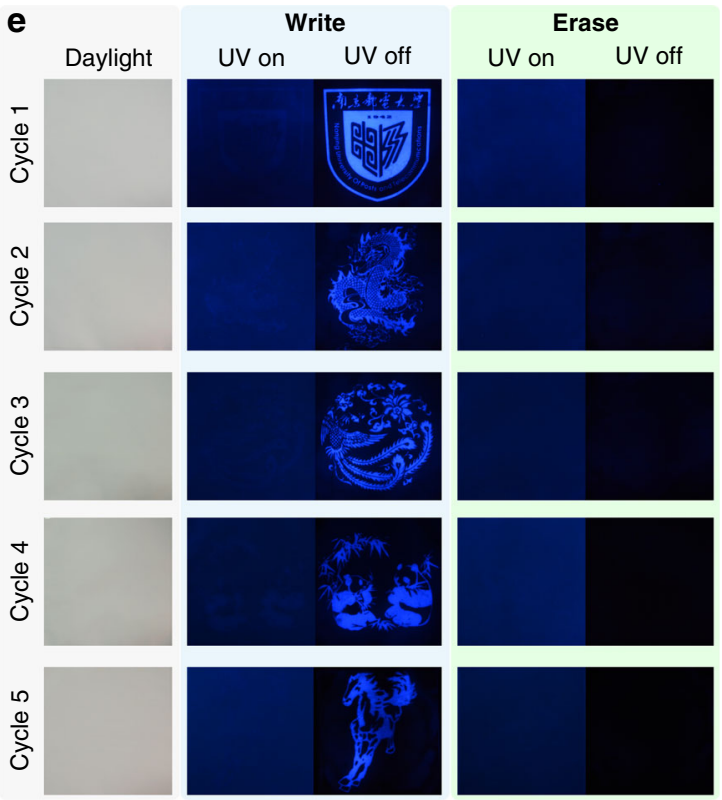

Fig. 5 Water-jet rewritable encryption papers. a Preparation of the rewritable encryption paper using pure water as ink. b Phosphorescence spectra of rewritable paper after writing and erasing. c Reversibility of the rewritable paper. d Photographs of the pattern under daylight and after the removal of 254 nm UV light. e Photographs taken during five cycles of the write/erase processes under daylight, UV light (UV-on), and after removal of the excitation (UV-off).

organic afterglow materials with PhQYs and lifetimes up to $46.1 \%$ and $1.67 \mathrm{~s}$, respectively. The principle of this approach, which involves using active host to sensitize the triplet exciton population via Dexter energy transfer for strong phosphorescence and water implement to rigidify the matrices through $\mathrm{H}$-bonding networks to suppress the non-radiative decays for high-efficient emission, provides a universal and convenient access to design highly luminescent and efficient OURTP observable by naked eyes under ambient conditions. Moreover, with the unique waterresponsible OURTP mechanism, we develop a rewritable lifetimeencryption paper using CT5 as substrate and water as ink for the high resolution water-jet printing of any patterns that can last for 1 month and be erased by DMSO vapor conveniently in $15 \mathrm{~min}$. Although there is still much to be learned regarding the exact mechanism in triplet excited state sensitization and stabilization, this host sensitization/water rigidification strategy would hold substantial promise for advancing the development of highperformance blue/deep-blue OURTP materials with high efficiency, strong brightness and long lifetime simultaneously and may inspire future innovative applications with the stimuliresponsible phosphorescent features.

\section{Methods}

Preparation of CA-based composites. CA-based deep-blue OURTP composites including CT, CI, CTP, and CP were prepared in a standard procedure. Take CT5 as a typical example. First, $5 \mathrm{mg}$ TMA was dissolved in $5 \mathrm{~mL}$ deionized water to obtain a $1.0 \mathrm{mg} / \mathrm{mL}$ aqueous solution of TMA. Second, to the $1 \mathrm{~mL}$ TMA solution was added $0.2 \mathrm{~g} \mathrm{CA}$, and the mixture was ultrasonicated at room temperature for $10 \mathrm{~min}$. Finally, the mixture was dried by removing the solvent of water under vacuum at $40{ }^{\circ} \mathrm{C}$ for $24 \mathrm{~h}$ to get the target composite of CT5-0 with TMA weight content of 5\%. To obtain CT5-20 with 20 wt $\%$ water, $25 \mu \mathrm{L}$ water was added to the 0.1 g CT5-0 followed by ultrasonication at room temperature for $10 \mathrm{~min}$.

Fabrication of the rewritable lifetime-encryption paper. $500 \mathrm{mg} \mathrm{CA}$ and $5 \mathrm{mg}$ TMA were dissolved in $5 \mathrm{~mL}$ DMSO to afford the coating solution. This solution was uniformly coated on a filter paper and after drying at $100^{\circ} \mathrm{C}$ for $2 \mathrm{~h}$, the rewritable lifetime-encryption paper was obtained.

Water-jet printing of the rewritable paper. The desired pattern was printed by a commercially available printer (HP DeskJet 1111) driven by a computer using pure water as ink.

Erase of the rewritable paper. The pattern printed on the rewritable paper was erased using DMSO vapor generated by heating DMSO to $120^{\circ} \mathrm{C}$. The complete erase of the pattern costs about $15 \mathrm{~min}$.

\section{Data availability}

The data that support the findings of this study are available from the corresponding author upon reasonable request.

Received: 25 March 2020; Accepted: 21 August 2020; Published online: 23 September 2020 


\section{References}

1. Ahn, D. H. et al. Highly efficient blue thermally activated delayed fluorescence emitters based on symmetrical and rigid oxygen-bridged boron acceptors. Nat. Photon. 13, 540-546 (2019).

2. Lee, J. et al. Deep blue phosphorescent organic light-emitting diodes with very high brightness and efficiency. Nat. Mater. 15, 92-99 (2016).

3. Pashaei, B. et al. Polypyridyl ligands as a versatile platform for solid-state light-emitting devices. Chem. Soc. Rev. 48, 5033-5139 (2019).

4. Liu, Y. et al. All-organic thermally activated delayed fluorescence materials for organic light-emitting diodes. Nat. Rev. Mater. 3, 18020 (2018).

5. Yan, D. \& Evans, D. G. Molecular crystalline materials with tunable luminescent properties: from polymorphs to multi-component solids. Mater. Horiz. 1, 46-57 (2014).

6. An, Z. et al. Stabilizing triplet excited states for ultralong organic phosphorescence. Nat. Mater. 14, 685-690 (2015).

7. Xu, S., Chen, R., Zheng, C. \& Huang, W. Excited state modulation for organic afterglow: materials and applications. Adv. Mater. 28, 9920-9940 (2016).

8. Kenry, Chen,C. \& Liu, B. Enhancing the performance of pure organic roomtemperature phosphorescent luminophores. Nat. Commun. 10, 2111 (2019).

9. Hirata, S. Recent advances in materials with room-temperature phosphorescence: photophysics for triplet exciton stabilization. Adv. Opt. Mater. 5, 1700116 (2017).

10. Zhou, B. \& Yan, D. Simultaneous long-persistent blue luminescence and high quantum yield within $2 \mathrm{D}$ organic-metal halide perovskite micro/nanosheets. Angew. Chem. Int. Ed. 58, 15128-15135 (2019).

11. Liao, Q. et al. 9,9-dimethylxanthene derivatives with room-temperature phosphorescence: substituent effects and emissive properties. Angew. Chem Int. Ed. 59, 9946-9951 (2020).

12. Yuan, J. et al. Invoking ultralong room temperature phosphorescence of purely organic compounds through $\mathrm{H}$-aggregation engineering. Mater. Horiz. 6, 1259-1264 (2019)

13. Kabe, R. \& Adachi, C. Organic long persistent luminescence. Nature 550, 384-387 (2017).

14. Jinnai, K., Kabe, R. \& Adachi, C. Wide-range tuning and enhancement of organic long-persistent luminescence using emitter dopants. Adv. Mater. 30, 1800365 (2018).

15. Lin, Z., Kabe, R., Wang, K. \& Adachi, C. Influence of energy gap between charge-transfer and locally excited states on organic long persistence luminescence. Nat. Commun. 11, 191 (2020).

16. Mao, Z. et al. The methylation effect in prolonging the pure organic room temperature phosphorescence lifetime. Chem. Sci. 10, 179-184 (2019).

17. Ma, X., Wang, J. \& Tian, H. Assembling-induced emission: an efficient approach for amorphous metal-free organic emitting materials with room temperature phosphorescence. Acc. Chem. Res. 52, 738-748 (2019).

18. Hirata, S. et al. Efficient persistent room temperature phosphorescence in organic amorphous materials under ambient conditions. Adv. Funct. Mater 23, 3386-3397 (2013).

19. Li, D. et al. Amorphous metal-free room-temperature phosphorescent small molecules with multicolor photoluminescence via a host-guest and dualemission strategy. J. Am. Chem. Soc. 140, 1916-1923 (2018).

20. Hirata, S. et al. Reversible thermal recording media using time-dependent persistent room temperature phosphorescence. Adv. Opt. Mater. 1, 438-442 (2013).

21. $\mathrm{Su}, \mathrm{Y}$. et al. Ultralong room temperature phosphorescence from amorphous organic materials toward confidential information encryption and decryption. Sci. Adv. 4, eaas9732 (2018).

22. Ma, X., Xu, C., Wang, J. \& Tian, H. Amorphous pure organic polymers for heavy-atom-free efficient room-temperature phosphorescence emission. Angew. Chem. Int. Ed. 57, 10854-10858 (2018).

23. Adam, N. Water cinderella ingredient of concrete. Concr. Int. 22, 66-71 (2000).

24. Arrachart, G. et al. Silylated melamine and cyanuric acid as precursors for imprinted and hybrid silica materials with molecular recognition properties. Chem. Eur. J. 15, 6279-6288 (2009).

25. Gong, Y. et al. Crystallization-induced dual emission from metal- and heavy atom-free aromatic acids and esters. Chem. Sci. 6, 4438-4444 (2015).

26. Gu, L. et al. Colour-tunable ultra-long organic phosphorescence of a singlecomponent molecular crystal. Nat. Photon. 13, 406-411 (2019).

27. $\mathrm{Li}, \mathrm{Q}$. et al. Induction of long-lived room temperature phosphorescence of carbon dots by water in hydrogen-bonded matrices. Nat. Commun. 9, 734 (2018).

28. Yang, X. \& Yan, D. Strongly enhanced long-lived persistent room temperature phosphorescence based on the formation of metal-organic hybrids. Adv. Opt. Mater. 4, 897-905 (2016).

29. Zhao, W. et al. Boosting the efficiency of organic persistent room-temperature phosphorescence by intramolecular triplet-triplet energy transfer. Nat. Commun. 10, 1595 (2019).
30. Jin, J. et al. Thermally activated triplet exciton release for highly efficient trimode organic afterglow. Nat. Commun. 11, 842 (2020).

31. Yang, Z. et al. Boosting quantum efficiency of ultralong organic phosphorescence up to $52 \%$ via intramolecular halogen bonding. Angew. Chem. Int. Ed. https://doi.org/10.1002/anie.202007343 (2020).

32. $\mathrm{Li}, \mathrm{H}$. et al. Stimuli-responsive circularly polarized organic ultralong room temperature phosphorescence. Angew. Chem. Int. Ed. 59, 4756-4762 (2020).

33. Nakamura, K., Hatakeyama, T. \& Hatakeyama, H. Studies on bound water of cellulose by differential scanning calorimetry. Text. Res. J. 51, 607-613 (1981)

34. Sun, J. et al. Charge-transfer exciton manipulation based on hydrogen bond for efficient white thermally activated delayed fluorescence. Adv. Funct. Mater. 30, 1908568 (2020).

35. $\mathrm{Wu}, \mathrm{H}$. et al. Molecular phosphorescence in polymer matrix with reversible sensitivity. ACS Appl. Mater. Interfaces 12, 20765-20774 (2020).

36. Zhou, B. \& Yan, D. Hydrogen-bonded two-component ionic crystals showing enhanced long-lived room-temperature phosphorescence via TADF-assisted Forster resonance energy transfer. Adv. Funct. Mater. 29, 1807599 (2019).

37. Gao, R. \& Yan, D. Layered host-guest long-afterglow ultrathin nanosheets: high-efficiency phosphorescence energy transfer at 2D confined interface. Chem. Sci. 8, 590-599 (2017)

38. Ma, Y. et al. Dynamic metal-ligand coordination for multicolour and water-jet rewritable paper. Nat. Commun. 9, 3 (2018)

39. Khazi, M. I., Jeong, W. \& Kim, J. Functional materials and systems for rewritable paper. Adv. Mater. 30, 1705310 (2018).

\section{Acknowledgements}

This work is supported by the National Natural Science Foundation of China (21772095, 91833306, 21674049, 61875090, 91733302, and 61935017), Key giant project of Jiangsu Educational Committee (19KJA180005), the fifth 333 project of Jiangsu Province of China (BRA2019080), China Postdoctoral Science Foundation (2020M671460), Jiangsu Planned Projects for Postdoctoral Research Funds (2020Z137), 1311 Talents Program of Nanjing University of Posts and Telecommunications (Dingshan) and the Science and Technology Development Fund Macao SAR (File no. FDCT-0044/2020/A1)

\section{Author contributions}

S.X., W.W., and R.C. conceived the experiments. S.X., C.Z., W.H. G.X., and R.C. wrote the manuscript. S.X., W.W., and J.Z. were primarily responsible for the material preparation. W.W., J.Z., H.L., and S.W. measured the photophysical properties and structure characterizations. S.W. performed the theoretical calculations. C.S. measured the Raman spectra. All authors contributed to the discussion of the results.

\section{Competing interests}

The authors declare no competing interests.

\section{Additional information}

Supplementary information is available for this paper at https://doi.org/10.1038/s41467020-18572-9.

Correspondence and requests for materials should be addressed to R.C., G.X. or W.H

Peer review information Nature Communications thanks Hengwei Lin, Dongpeng Yan and Hui $\mathrm{Xu}$ for their contribution to the peer review of this work. Peer reviewer reports are available.

Reprints and permission information is available at http://www.nature.com/reprints

Publisher's note Springer Nature remains neutral with regard to jurisdictional claims in published maps and institutional affiliations.

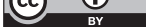

Open Access This article is licensed under a Creative Commons Attribution 4.0 International License, which permits use, sharing, adaptation, distribution and reproduction in any medium or format, as long as you give appropriate credit to the original author(s) and the source, provide a link to the Creative Commons license, and indicate if changes were made. The images or other third party material in this article are included in the article's Creative Commons license, unless indicated otherwise in a credit line to the material. If material is not included in the article's Creative Commons license and your intended use is not permitted by statutory regulation or exceeds the permitted use, you will need to obtain permission directly from the copyright holder. To view a copy of this license, visit http://creativecommons.org/ licenses/by/4.0/

(c) The Author(s) 2020 